\title{
Note sur une restauration effectuée dans une ville maghrébine: entre pastiche et authenticité
}

\author{
Mohammed Bouziane \\ Université des Sciences et de la Technologie d'Oran
}

\begin{abstract}
RESUMEN. Este artículo examina una operación de restauración efectuada en Argelia, precisamente en Mascara, en el año 2001. En efecto, el texto concierne la restauración de dos edificios que datan del decimoctavo siglo (estilo otomano). Básicamente, la reflexión trata de analizar la operación considerándola en su contexto real, en sus circunstancias excepcionales (guerra civil en Argelia, tradición arquitectónica, etc.). En este examen intentamos demostrar que lo que podría ser considerado como una falsificación en Europa, en Argelia, o en cualquier otro país islámico, puede ser considerado como una 'restauración auténtica'. El joven arquitecto (Sr. SADEG), a pesar de las dificultades, ha realizado un trabajo coherente si se consideran las condiciones excepcionales y el contexto que caracterizaron ese periodo (década negra de los años noventa).

Palabras clave: Terrorismo, restauración, restitución, estilo otomano, Abdelkader, Entrestaurierung, Argelia, Magreb, Mascara.

RESUME. Dans cet article on a essayé d'analyser une opération de restauration effectuée en 2001 à Mascara (Algérie). En effet, cet écrit concerne la restauration de deux édifices datant du dix-huitième siècle (style ottoman). Il s'agit grosso modo, de situer l'opération dans son vrai contexte et de déterminer les circonstances qui l'ont marquée (Guerre civil, tradition architectonique, ...etc.). Aussi, par cette réflexion on a essayé de prouver, plus ou moins, que ce qui pourrait être considéré en occident comme une falsification, en Algérie, peut être considéré comme une 'restauration authentique', celle que l'on appelle 'la restauration permanente'. On peut estimer que le jeune architecte (M. SADEG), malgré les difficultés, a réalisé un travail cohérent en prenant en considération le contexte difficile ainsi que les conditions qui ont marqué cette période( décennie noire du terrorisme islamiste).

Mots clé: Terrorisme, restauration, restitution, ottoman style, Abdelkader, Dérestauration, Algérie, Maghreb, Mascara.
\end{abstract}

\section{CONTEXTE DE LA RESTAURATION (OU LA RESTAURATION COMME ARME ANTITERRORISTE).}

Durant la décennie noire, le terrorisme s'est attaqué férocement à tout ce qui symbolisait l'état algérien moderne. Des écoles primaires, des usines, des commissariats, et des centres culturelspour ne citer que ceux-là - ont été brûlés, voire même complètement détruits. Pire encore, les symboles de l'islam traditionnel algérien, tolérant et généreux, se sont vus attaqués. Ainsi, les mausolées de plusieurs saints (wali) ont été la cible de bombes artisanales. Comme ce fut le cas du mausolée de Sidi Boumediène ${ }^{1}$ l'un des joyaux de l'architecture islamique universelle, qui a été partiellement endommagé par un engin explosif.

${ }^{1}$ W. \& G. MARÇAIS, Monuments Arabes de Tlemcen, Paris, 1903, pp. 230-239. 
Pour des raisons quel'on peut facilement comprendre, les administrateurs s'empressaient toujours de remettre en état, toujours dans la mesure du possible et non sans difficultés, les édifices d'utilité publique endommagés. De là, est née une logique rigoureuse de réparation des édifices endommagés par les bombes. C'était une logique qui était basée sur l'urgence, la rapidité, le pragmatisme, au détriment, bien sûr, de la qualité. Il s'agissait, aussi, d'éviter aux populations le traumatisme engendré par une guerre qui ne voulait pas annoncer son nom. C'était la guerre psychologique.

Dans un contexte marqué par cette guerre psychologique et spirituelle, l'identité nationale, entre autres valeurs, s'est vue remise en cause. L'Algérie, par conséquent, avait besoin de repères culturels et historiques. Les idéaux et les symboles de Novembre ne semblaient plus suffisants pour réconforter le peuple algérien dans ses convictions. C'est dans ces circonstances que l'Algérie et ses responsables politiques avaient besoin de réhabiliter ses symboles nationaux; ceux qui réconciliaient modernité et Islam, tolérance et religion. On avait besoin de puiser dans l'Histoire pour parer à cette déferlante islamiste.

Ainsi, le personnage de l'Emir Abd elKader ${ }^{2}$, célébré aujourd'hui comme le fondateur de la nation algérienne, est réapparu comme une icône de l'Algérie historique à la fois moderne et musulmane. Il s'est avéré nécessaire de lui redorer son blason à celui qui a combattu farouchement les généraux Bugeaud et Mac Mahon; celui

\footnotetext{
${ }^{2}$ Voir; P. AZAN, L'Emir Abd el-Kader (1808-1883), Paris, 1925. A.. Bellemare, Abd el-Kader. Sa Vie Politique et Militaire, Paris, 1854. W. BLUNT, Desert Hawk. Abd el-Kader and the French Conquest of Algeria, Londres, 1947. C. H. CHURCHILL, La Vie d'Abdel Kader. Ex-Sultan des Arabes de l'Algérie, Londres, 1867.
}

qui a été décoré de l'ordre de Pie IX et qui a reçu la grand-croix de la Légion d'honneur.

C'est dans ce contexte de postterrorisme, marqué par une recherche de repères, et sur une décision politique, qu'une enveloppe budgétaire très importante a été débloquée par le ministère de la culture pour la réhabilitation et la restauration de l'ancien Quartier Général de l'émir Abd el-Kader ainsi que de son Tribunal.

Longtemps délaissé, le Quartier Général de l'émir à Mascara était livré à lui même. Son tribunal, qui se trouve juste à côté et dont la dernière fonction consistait à abriter un salon de thé, a été considérablement détruit par le séisme qui a secoué la ville de Mascara en 1995. Bref, rien, en tout cas, ni personne ne présageait que ces deux édifices, qui avaient connu une histoire tumultueuse et riche, allaient être sauvés d'une disparition lente mais certaine.

\section{HISTORIQUE ET PASSÉ POLITIQUE D'UN LIEU HAUTEMENT EMBLÉMATIQUE.}

Ce qui est considéré de nos jours comme le quartier général de l'émir est, en fait, un édifice construit par les Ottomans. En effet, lorsque Mascara était la capitale du "beylik" du Couchant, le bey Mohammed elKabir ordonna sa construction aux alentours de 1789 dans le but d'abriter ce qui devait être son palais. Mais, pratiquement, ça n'a pas été ainsi puisque ce dernier avait déménagé à Oran une fois celle-ci libérée des Espagnols en 1792. Jusqu'à l'occupation d'Alger par les Français (1830) et la disparition qui s'en suivit du pouvoir ottoman, ce bâtiment n'aurait été occupé que par un piètre représentant du pouvoir ottoman. D'où son attribution historique et surtout populaire à l'émir $A b d$ el-Kader qui en a fait son quartier général, lorsqu'il déclara la guerre sainte (le djihad) aux 
Français. Une fois la guerre terminée et l'émir arrêté, ce sont les Français qui vont l'occuper à leur tour aux alentours de 1848 pour en faire le quartier général de l'armée française dans la ville en l'adaptant, biensûr, aux exigences des nouvelles fonctions. En 1949, quand il fut déclaré "Monument Historique" par les autorités coloniales, il constituait la résidence de l'administrateur civil. Entre temps, il avait subi plusieurs modifications, des fois capricieuses, autres fois justifiées. C'est à partir de l'indépendance (1962) que le sort de l'édifice devient très triste. Malgré sa dépendance de l'A.P.C, cet édifice fut quasiment abandonné pour n'avoir eu aucune fonction ou affectation. Ce qui l'a laissé à la merci des "aléas climatiques" et des squatters qui en ont fait un immeuble de rapport. L'histoire du tribunal n'en est pas moins agitée et triste. En effet, ce qui était un salon de thé, il y a peu de temps, était à l'époque coloniale le Centre de Bienfaisance Européen. Et ce, après avoir été successivement un tribunal militaire à l'époque des Ottomans et un tribunal, plus civil que militaire, durant le règne de l'émir Abd el-Kader (1832-1847).

L'histoire de ces deux édifices, pleine de tumultes, à l'image du lieu, laisse présager qu'une éventuelle restauration serait très problématique. Objet de convoitise de tous les pouvoirs qui se sont succédés dans cette petite ville de l'ouest algérien, ils ont toujours été associés à une certaine connotation politique, à une certaine manifestation de l'autorité. Au fil du temps, ces deux édifices ont acquiert, malgré eux, une condition symbolique patente représentant un certain exercice du pouvoir qui a caractérisé surtout celui $\mathrm{d}^{\prime} A b d$ el-Kader (séparation des pouvoirs). Le site-Place des Martyrs, ex-place Mogadordans lequel ils se trouvent y est pour beaucoup. Situés dans ce qui a été le centre politique de la cité médiévale, créée pour des besoins militaire au XV ème, ces édifices abritaient tous les pouvoirs que sont l'exécutif, le judiciaire et "le constitutionnel": La grande mosquée zianide, se trouvant vers l'ouest à quelques mètres seulement du tribunal, assumait le rôle du contrôle constitutionnel par le biais des ulémas qui veillaient à la conformité des lois et des jugements par rapport au coran et la sunna ${ }^{3}$.

Les Français en s'appropriant cet espace et en imposant le "style $d u$ vainqueur" ${ }^{\prime}$ ont compris, dès les premiers jours de l'occupation de cette ville, qu'ils allaient s'approprier tout le pouvoir et faire comprendre aux "indigènes" qu'ils étaient les nouveaux maîtres du pays. Cependant ils n'avaient pas accordé à ces deux édifices (le quartier général et le tribunal) la même attention qu'ils avaient accordée à la grande mosquée. Leur attitude en premier temps était dictée par une logique avant tout militaire.

En créant une rue qui relie la place des Martyres (ex-place Mogador) avec une esplanade -Place l'Émir Abd el-Kader- qui se trouve plus au nord et dont les dimensions avaient été calculées pour permettre l'alignement d'un bataillon, les ingénieurs français ont défiguré une première fois le tribunal dont la presque moitié (occidentale) était démolie pour disparaître définitivement afin de permettre le passage de cette rue. Il a été, une deuxième fois, fortement défiguré lorsque les responsables du Centre de Bienfaisance décidèrent de recouvrir le patio du tribunal pour gagner de l'espace.

\footnotetext{
${ }^{3}$ H.H. Hirèche, «L'Emir Abdelkader et la Modernité Occidentale au XIXème siècle», Le Quotidien d'Oran, juillet, 2003.

Cf. http://dzlit.free.fr/emaek.html (dernière visite 13 octobre 2005).

4 "Style du Vainqueur", expression utilisée par le général Dolot, «l'Architecture Moderne à Tunis», Revue Tunisienne, 1920, pour décrire le style de l'architecture qui a été produite durant les premières années de la colonisation française à Tunis.
} 
Des deux édifices restaurés, le tribunal est sûrement le plus déformé. Le quartier général n'a pas été ménagé non plus.

Le rajout du balcon d'honneur sur la façade principale du quartier général, élément classique de l'architecture européenne très utilisé dans la rive nord de la méditerranée, notamment en France d'où provenaient la grande majorité des colons, est une altération qui n'est légitimée par rien. De même que pour les moulures en forme de chaîne de besace qui ont en altéré l'esprit et constituent de ce fait une falsification outrageante. La conviction selon laquelle l'art contemporain européen était sans conteste supérieur aux expériences antérieures et, par conséquent, digne de substituer aux œuvres arabes (indigènes) les siennes propres détermina une opération qui fut proclamée "restauration", mais qui était en fait une totale transformation du monument. L'idéal néo-classique, dont se sont inspirés les architectes qui ont ajouté les moulures, porta à rejeter sans appel la production de ce qui représentait pour eux le Moyen Age. L'incurie et un total mépris ont failli entraîner une totale métamorphose de cet héritage voire même sa disparition...

Mais"S'il y a une liaison évidente entre le niveau historique et idéologique et le niveau architectural, l'analyse de l'architecture ne peut se présenter comme une projection simpliste de l'histoire sur l'espace et la forme architecturale $^{\prime \prime}$.

En effet, il est nécessaire de noter que l'appréhension d'une ville ou d'un lieu à travers les seuls arguments historiques risque d'aboutir à des jugements fermés: la ville "indigène" était inadaptée aux nouveaux modes de vie, par exemple. Les

${ }^{5}$ J.J. Deluz, L' Urbanisme et l'Architecture d'Alger, Liège, 1988, p. 7. transformations des sociétés, les révolutions, les mutations sociales, l'évolution des techniques qui s'y superposent, exigent un dépassement de ce niveau primaire. Paradoxalement, un examen purement architectural des formes du cadre bâti pourrait-il aider à cette appréhension. L'objet architectural jouit d'une vie propre plus ou moins riche en capacité de survie bien qu'il est vrai que l'analyse du cadre bâti donne raison à un certain déterminisme historique, et que le décryptage des formes, quelle que prétende être l'objectivité de l'histoire de l'art et de ses enchaînements stylistiques, passe par l'analyse des conflits idéologiques.

La complexe dialectique entre le progrès technique et le progrès social, que revendiquaient les interventions coloniales, est illustrée par l'exemple d'Haussmann: la rénovation répressive de l'ancien Paris (l'encerclement des îlots, les boulevards "policiers", l'éjection des classes populaires hors des quartiers privilégiés) se superpose à des mesures de progrès technique (hygiène, circulation) irréversibles. Que les raisons de ces progrès fussent purement spéculatives n'empêchera pas les architectes et les urbanistes modernes de s'y référer ${ }^{6}$.

Pour revenir à l'objet de cette réflexion, on constate qu'à l'idéologie coloniale, quel que fût le contenu de son discours (supériorité de l'art du vainqueur, logique militaire), des mesures de progrès technique se sont superposées (Transfert de know-how). C'est pour cette raison qu'on peut supposer que, des fois, les interventions françaises étaient justifiées et plus ou moins légitimes : Le principal argument étant le discours hygiéniste qui a tant caractérisé l'architecture et l'urbanisme de l'époque coloniale. L'édifice, n'étant pas prévu pour abriter le quartier général d'une

\footnotetext{
${ }^{6}$ J.J. DELUZ, ibidem, p. 8.
} 
armée moderne, a dû céder aux lacérations que lui ont infligé, entre autres, le temps, l'usage intense et les exigences du progrès technique (égouts, assainissement, dégagement des circulations). Ainsi, l'armée française a dû consolider le plancher de l'étage en remplaçant l'ancienne structure traditionnelle par des voûtains de briques, plus solides de point de vue structurel mais peu adaptés au climat. Même Cotereau, cité par J.J. Deluz ${ }^{7}$, paraît s'opposer à cette méthode. Celui-ci a laissé, dans les passages où l'idéologie "d'extrême droite" n'entache pas son discours, de bonnes analyses de la maison d'Alger, et en particulier de sa construction:

“... Sur un plancher reposaient alternativement une couche de pierraille, une couche de terre, enfin un enduit longuement damé. Tout cela atteignait une épaisseur considérable. Aussi, aujourd'hui encore, voiton ces vieilles terrasses, absolument imperméables, ne donner lieu à des gouttières qu'après réparations, aux parties réparées. Elles réalisaient aussi, du fait de leur épaisseur, un isolant thermique absolument remarquable. A ce point de vue, les terrasses modernes, trop minces, ne sont trop souvent, malgré leurs voûtelettes de briques ou leurs dalles de béton, que de véritables contrefaçons. Autant, dans les vieilles maisons turques, les pièces sont fraîches l'été, autant elles constituent dans les nôtres de véritables étuves..."

La grande mosquée, déclaré "Monument historique" dès 1919, a été quant à elle relativement ménagée. Une telle épargne de la part des architectes, et des ingénieurs ne s'expliquait qu'eu égard à des valeurs extrinsèques au monument telles que le caractère sacré de l'œuvre avec tout ce qui s'y attachait en fait de dévotions et de traditions. Il s'agit là d'un cas où le nouveau avait été introduit, à chaque fois, à petites doses dans l'ancien sans en

\footnotetext{
${ }^{7}$ J.J. DeLUZ, ibidem, p. 24.
}

corrompre l'âme et sans constituer une flagrante altération. On peut estimer qu'une exploration minutieuse pourrait dater, avec exactitude, chaque apport nouveau (période zianide, période turque, époque coloniale).

Avec la colonisation française, il a fallu parvenir à l'époque du gouverneur Jonnart (1898-1900, 1903-1911, 1919...), qu'on a suspecté d'indigénophilie, pour qu'on se tournât vers le passé comme vers une réserve culturelle qu'il importait de défendre contre les mutilations et les saccages arbitraires. Mais Lyautey disait:

“... Nos protégés ont compris le génie de notre race en nous regardant restaurer leurs monuments..."

La France s'est rendue compte du danger politique qui pouvait résulter de la destruction d'une structure sociale basée sur des formes ancestrales d'habitat et de coutumes urbaines ${ }^{9}$. On peut aussi invoquer la signification symbolique que peuvent générer quelques gestes de sauvegarde et de restauration. La France aurait une bonne image, celle d'une France protectrice, préoccupée par les traditions et respectueuses des coutumes locales. C'est ainsi, que ces édifices furent déclarés "Monuments Historiques" en 1949 soit seulement treize ans avant l'indépendance et cinq avant le déclenchement de la révolution...

\section{LA RECONSTRUCTION ET LE CARACTÈRE EXCEPTIONNEL DE L'INTERVENTION.}

L'état ruiniforme dans lequel se trouvait le tribunal de l'émir, conséquence

\footnotetext{
${ }^{8}$ Général LyAUTEY, «Lettre datée du 05 juillet 1917», l'Art et les Artistes, numéro spécial: Le Monde Artistique, 1916-1917, cité par J.F. BEGUIN, Arabisances et Tracés Urbains en Afrique du Nord, Paris, 1983.

9 J.F. Beguin, Arabisances et Tracés Urbains en Afrique du Nord, Paris, 1983.
} 
directe de la secousse tellurique de 1995, suggérait que le débat sur la restauration tournerait autour de la reconstruction: si elle devait être "une copie" ou "un dérivé", une "reconstruction" o une "rénovation", et si une reconstruction pouvait constituer une "falsification".

Des documents, issus des congrès internationaux et des lois de patrimoine des nations occidentales (ICOMOS), émerge un principe fondamental de la discipline de la restauration architectonique qui, avec l'extension du concept de Monument à celui de Document, exclut dogmatiquement toute intervention de reconstruction qui pourrait adopter la formule "où c'était et comme c'était" chère aux restaurateurs romantiques et historicistes du siècle passé. L'édifice obtenu après l'intervention se configurerait comme une copie, ou mieux, une "falsification": d'ailleurs, pour les historiens romantiques, toute restauration est une falsification, un moyen de remplacer le monument par un sosie artificiel et sans expression. Mais le concept de "falsification" se réfère ici uniquement au concept de "fraude", dans le sens où on ne témoignerait pas de notre temps de façon conforme et adéquate aux technologies contemporaines les plus avancées et les formes d'art qui se mettent en rapport avec ces technologies ${ }^{10}$.

Le tribunal de l'émir, comme document, est un original qui a été irrémédiablement détruit. Mais la perte du support matériel d'un document peut être substituée par un témoignage, un peu comme on authentifierait la copie d'un document en papier. Dans ce cas, c'est la communauté mascaréenne qui est la plus autorisée a émettre le "certificat de

-

${ }^{10}$ G. CRISTINELLI, «El proyecto de restauración y el concepto de monumento», Congreso Internacional de Restauración: "Restaurar la memoria", Valladolid 1998. Actas, Valladolid, 1998. p. 171. conformité" d'une nouvelle construction. Et c'est elle -et avec elle toute la société algérienne- qui veut aujourd'hui, en laissant à côté les "inutiles" controverses conceptuelles, que ce lieu soit reconstruit exactement comme il était auparavant ou du moins le plus semblable possible à celui qui a été détruit. En effet, le tribunal de l'émir est de ces ouvrages et ensembles qui ont pour une collectivité donnée une valeur irremplaçable de symbole qu'il faut maintenir à tout prix: la reconstruction, si laborieuse, des cathédrales de Reims et de Varsovie suffirait à nous le faire saisir, au cas où nous l'aurions oublié. Mais il faut noter une chose. Il s'agit d'une volonté de restitution intégrale qui va bien au-delà de la restauration prudente: “Un symbole doit être complet, explicite, éloquent pour satisfaire la conscience collective, et les impératifs scientifiques passent alors vite au second plan"11. C'est dans cette logique seule que la thèse de la particularité de l'intervention (la Reconstruction) a été - et est-acceptée.

\section{“DÉRESTAURATION”, AUTHENTICITÉ ET TRADITION ARCHITECTONIQUE.}

En reconstruisant le tribunal, l'architecte lui a restitué sa disposition spatiale initiale, à savoir le patio autour duquel s'organisait les espaces de cet édifice à l'époque de l'émir et que les responsables du Centre de Bienfaisance Européen, à l'époque coloniale, avaient décidé d'éliminer pour gagner de l'espace. Or, la Charte de la restauration de Venise (1964) comporta une notion révolutionnaire en énonçant le principe de la conservation intégrale des divers stades historiques qui ont marqué une œuvre, en le justifiant par la possibilité (pour les temps à venir, qui seront dotés de ressources et d'instruments

11 A. CHASTEL, «Patrmoine monumental», $C D$ ROM Universalis multimédia, version 6, 2000. 
plus perfectionnés que ceux d'aujourd'hui) de découvrir des valeurs qui échappent à l'analyse actuellement possible. L'architecte, avec cette décision, justifiée par l'exigence archéologique, a condamné le témoignage historique et artistique de la précédente intervention en éliminant le plancher apocryphe qui couvrait le patio original. Il a, pour ainsi dire, opté pour le parti pris de la "dérestauration". Là encore on est en droit de se demander si avec cette attitude on a restitué à l'œuvre son authenticité, objectif premier de toute opération de restauration.

La "dérestauration", que la langue allemande dénomme avec une nuance plus exacte "Entrestaurierung", se définirait comme l'élimination des éléments apocryphes, hérités des interventions précédentes ${ }^{12}$. Pourtant, les expériences tentées ont toutes amené à reconnaître, ce qui est logique, que soustraire les compléments ajoutés ultérieurement n'équivaut pas nécessairement à restituer son authenticité à l'original ${ }^{\left[{ }^{13}\right]}$. Pour comprendre un petit peu l'attitude de l'architecte, qui revendique l'authenticité de l'œuvre, il faudrait retenir la thèse qu'expose le professeur G. Cristinnelli ${ }^{14}{ }^{14}$, qui distingue entre Authenticité, comme reflet de la substance du monument et qui doit être persécutée à outrance par le restaurateur, et Identité, comme la partie du monument qui pourrait être subrogée. Le patio dans ce sens se révèlerait être la substance du monument et de tout édifice de l'époque. En lui restituant son patio l'architecte, $M$. Sadeq, a rétabli l'édifice dans ses droits.

On pourrait expliquer cette restitution par une autre définition. Celle

\footnotetext{
-

${ }^{12}$ C. Di MATTEO, «Restauration des œuvres d'art», CD-ROM Universalis multimédia, version 6, 2000.

13 C. Di MATTEO, idem.

14 Professeur à l'Institut Universitaire d'Architecture de Venise.
}

avancée par $A$. Chastel ${ }^{15}$ qui parle d'une restauration permanente lorsque il évoque les temples japonais de Kyoto. Ces monuments sacrés sont, pendant des siècles, maintenus à l'identique à travers un nombre incroyable de destructions dues aux séismes et aux incendies, dont on élimine soigneusement toute trace. La transcendance du symbole entraîne ici en somme, la restauration occulte. La substance est, dans ce cas, la forme qui est maintenue à outrance, au détriment de la matière qui est substituée périodiquement, sans altérer la symbolique ni la compréhension des objets du passé. Sans doute, l'idée d'authenticité se trouve mieux définie en orient qu'en occident.

Les exemples dans la tradition architectonique islamique ne manquent pas. La Grande mosquée de Cordoue (Espagne) est, à ce titre, très évocatrice. Elle démontre, malgré les extensions, effectuées tout au long de deux siècles (entre 785 et 987), et les nombreuses nefs ajoutées, que l'unité originelle du lieu n'a pas été affectée. $\mathrm{Au}$ contraire, son effet est réaffirmé. La substance du monument, ou du moins, l'une de ses substances essentielles, est ici sa disposition spatiale ordonnancée en une grande salle hypostyle où la suprématie de l'ordre horizontal, assisté par l'armada de colonnes se répartissant dans les deux directions du plan horizontal, est incontestable. L'impression d'espace sans limites, obtenue par la répétition infinie des supports verticaux, est conservée à chaque opération d'extension.

En fin, on pourrait évoquer, à titre de dernier exemple, les koubbas maghrébines dont la pérennité de la forme qui les caractérise n'a été possible que grâce à la restauration permanente et occulte. Ce type d'édifice, apparu pour la première fois au

${ }^{15}$ Professeur au Collège de France. 
neuvième siècle à Kairouan, a maintenu à outrance sa forme pendant des siècles pour nous arriver avec l'aspect qu' on lui connaît de nos jours. Ici non plus, la problématique de l'authenticité n'a pas lieu d'être.

\section{LE QUARTIER GÉNÉRAL: LIEU, MATÉRIAUX UTILISÉS ET SUBSTANCE.}

L'état sanitaire du quartier général laissait conjecturer que sa restauration serait beaucoup moins problématique. Là, il n'était pas question de reconstruire mais de faire une restauration proprement dite.

L'exercice de restauration du quartier général s'est révélé plus judicieux, moins problématique et, en un sens, beaucoup plus "facile". En effet, on peut estimer que l'opération de restauration était appropriée avec le caractère documentaire du monument. A cela on peut avancer plusieurs raisons.

L'opus incertum, qui caractérise les appareils de ce monument, est toujours en vigueur dans les constructions typiques des environs, notamment dans le milieu rural. Ici, il n'est pas question de "fraude", ni dans le sens de "falsification", caractéristique des restaurations faites en Europe au siècle passé, ni dans le sens évoqué par $G$. Cristinelli. D'ailleurs, les maçons qui ont exécuté les travaux, sont ces mêmes artisans qui, avec les mêmes procédés d'il y a deux siècles, toutes considérations gardées, continuent de construire pour la masse populaire. Il faut noter tout de même qu'il aura fallu à l'architecte un effort pédagogique pour enseigner à ces artisans la méthode qu'on utilisait auparavant pour supporter le plancher de l'étage; celle qui avait "l'aval" de Cotereau et que la dalle en béton armée avait remplacée progressivement à partir des années soixante-dix. Aussi, il faut signaler qu'il aura fallu extraire d'une lointaine carrière fermée la chaux utilisée comme mortier dans ces deux constructions.
L'élimination des séparations qu'avaient introduites les squatters, qui occupaient la bâtisse, serait une "dérestauration" très appropriée et, par conséquent, "académique". Il n'aura pas fallu beaucoup de recherche pour déduire qu'il fallait débusquer ces éléments apocryphes de mauvaise facture.

Aussi, l'architecte a eu raison en ménageant les moulures en forme de chaîne de besace (à l'angle du monument) ainsi que balcon d'honneur rajouté (sur la façade principale). Témoins de l'intervention coloniale précédente, leur élimination n'aurait pas donné plus de crédibilité à la volonté de restitution historique. Au contraire, leur présence ne fait que renforcer l'identité de l'œuvre -dans le sens évoqué auparavant - et du lieu (l'explace Mogador, actuelle place des Martyrs). L'art français-et avec lui l'occupation française-bien qu'apocryphe, fait maintenant partie de l'histoire du monument. L'exigence historique, au détriment de la restitution du même nom, voudrait qu'on laisse vivant ce témoignage. La réflexion aurait été autre (dans le sens contraire), si la modification avait été très traumatisante et avait touché à la substance $\mathrm{du}$ monument (la disposition spatiale, l'austérité de la façade, intériorité de l'architecture, ...etc.) Ceci dit, on peut regretter que, pour des raisons purement techniques, la conservation du plancher colonial -témoignage architectonique du transfert du know-how occidental- n'ait pu avoir lieu.

Si l'on est étonné, en visitant cet édifice, de la somptuosité toute relative de l'architecture intérieure -elle nous rappelle étrangement l'architecture citadine d'Alger (La casbah) ainsi que les résidences qu'a édifiées la "nomenklatura" ottomane aux environs de la capitale - et totalement contradictoire avec l'esprit à la fois modeste et noble de l'émir, dont "le seul luxe 
vestimentaire, malgré son aisance matérielle, consistait en ses armes" ${ }^{16}$; Si l'on est étonné de l'absence de l'élément végétal, caractéristique essentielle des palais qu'a occupés l'émir durant son règne - notamment ceux de Médéa et de Miliana, également de facture turque - Lauthenticité de la conception (de l'organisation spatiale), des matériaux utilisés, de la main d'œuvre, ainsi que de l'environnement fait que cet édifice, tel qu'il se présente après la restauration, est authentique.

${ }^{16}$ C. H. Churchill, La Vie d'Abdel Kader, Alger, 1971. p. 49 . 


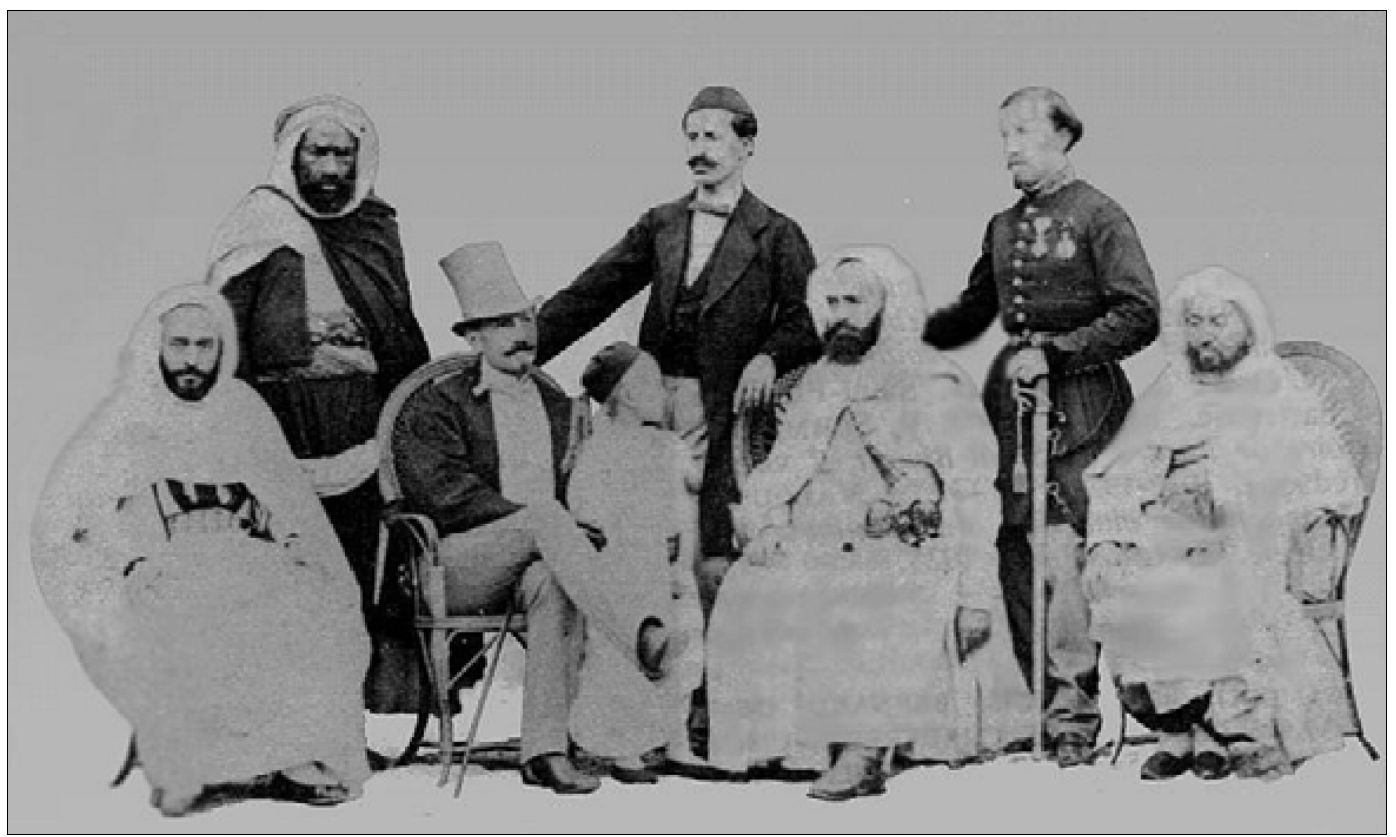

- Fig. 1. 1867, l'Émir avec à sa gauche le général Mac-Mahon, futur président de la République Française (Archives de la Bibliothèque Nationale de France).

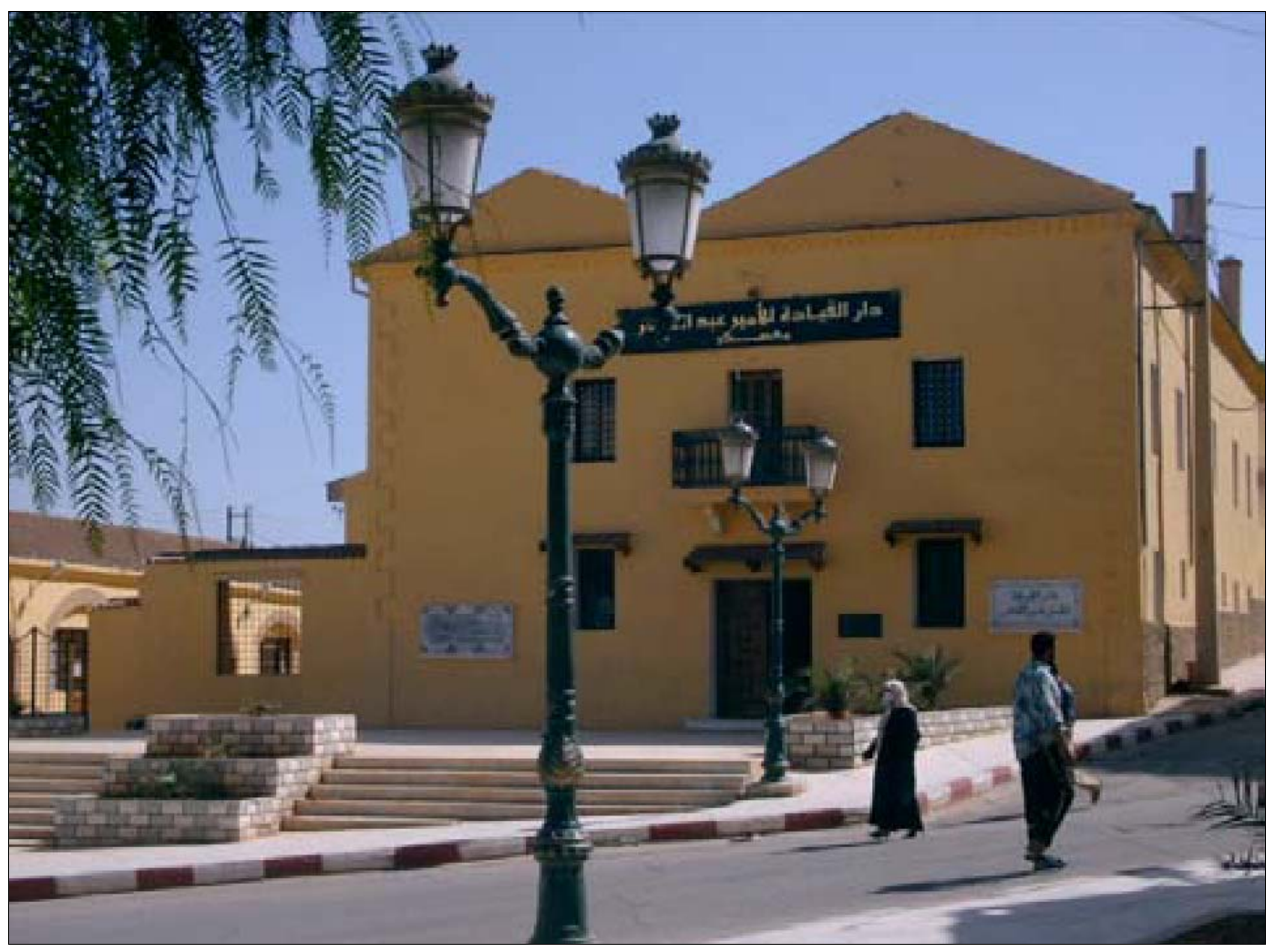

- Fig. 2. Quartier général de l'émir à Mascara, après restauration (M. Bouziane). 


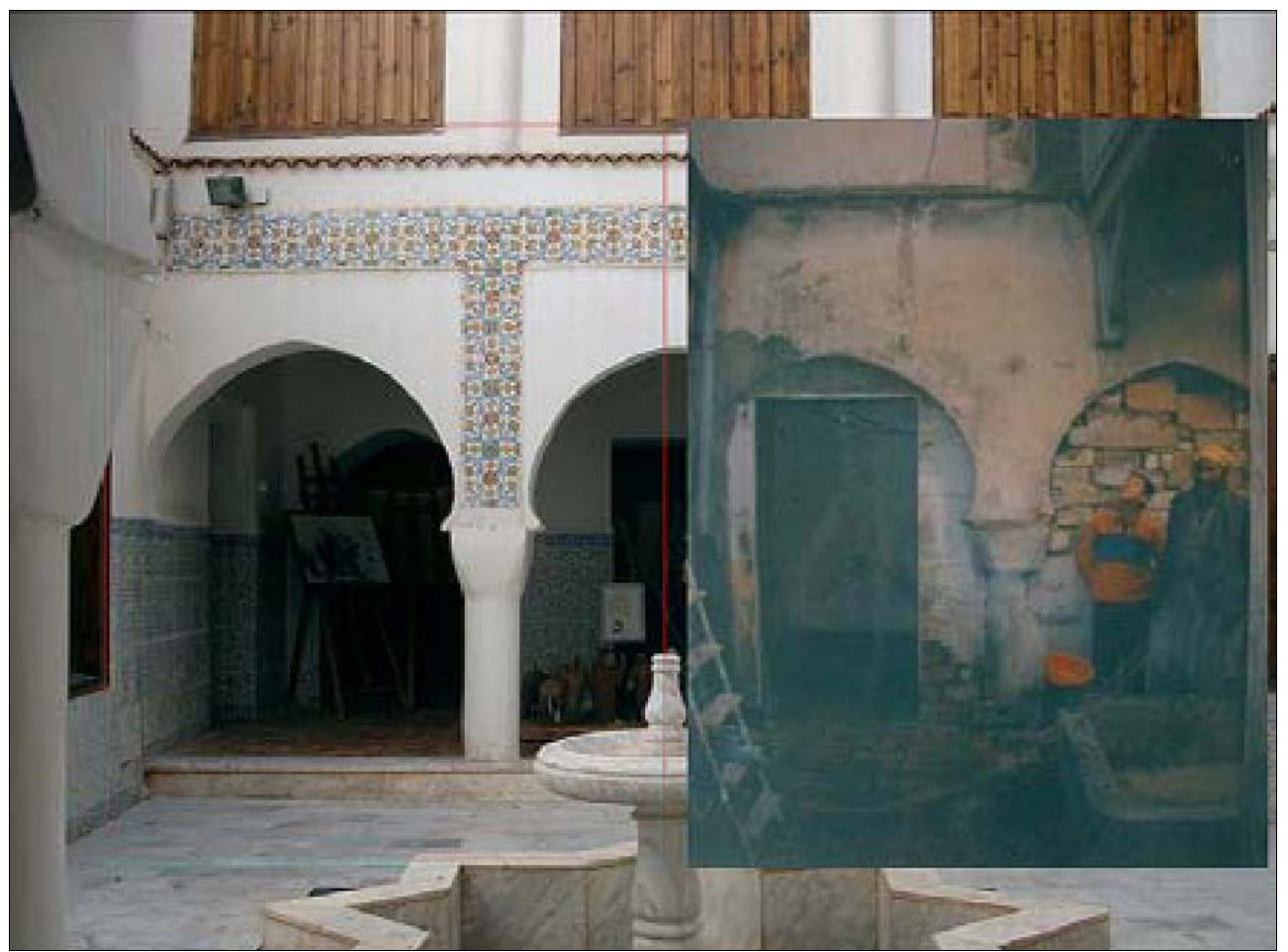

- Fig. 4. Patio restauré du quartier général. L'architecte a éliminé la passerelle "apocryphe" (en bois) introduite par les architectes de l'époque coloniale (M. Sadeg et M. Bouziane).
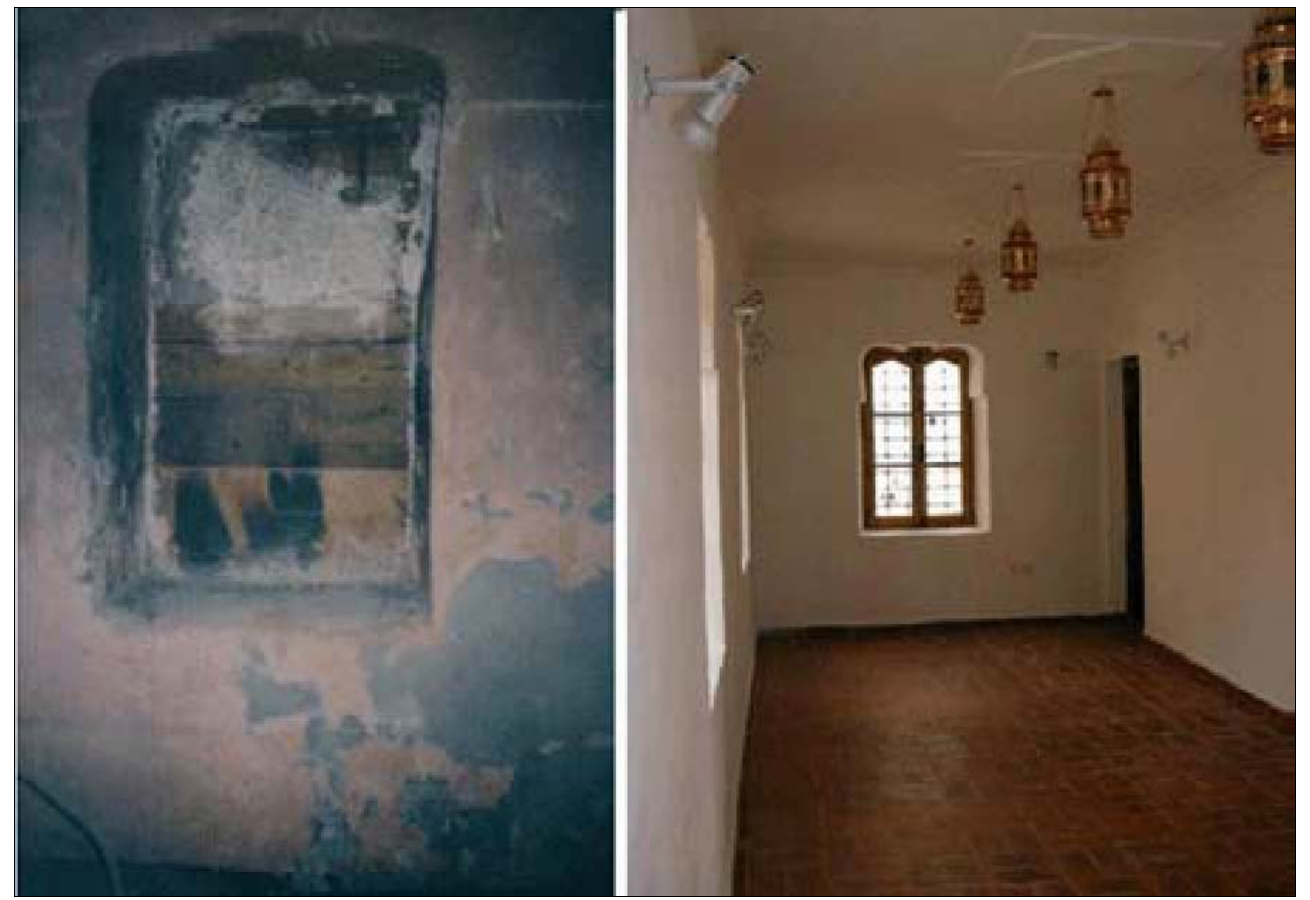

- Fig. 4. Une fenêtre typique du style ottoman restaurée. Tribunal (M. Sadeg et M. Bouziane). 


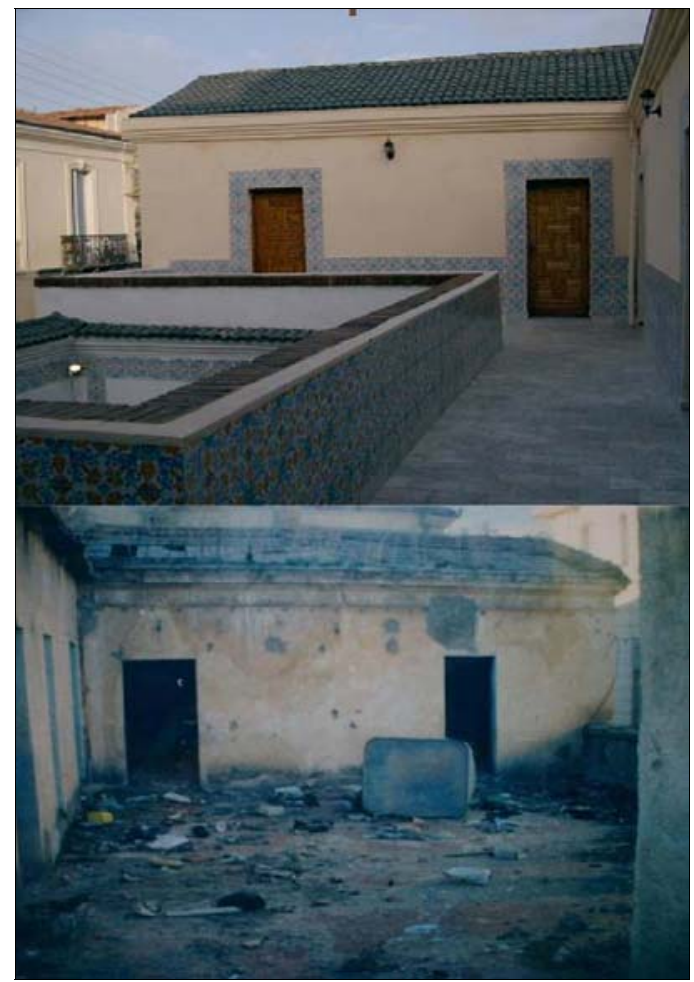

- Fig. 5. Patio du tribunal. Après et avant la restauration (M. Sadeg et M. Bouziane).

- Fig. 6. Quartier Général. Le péristyle après sa restauration (M. Bouziane).

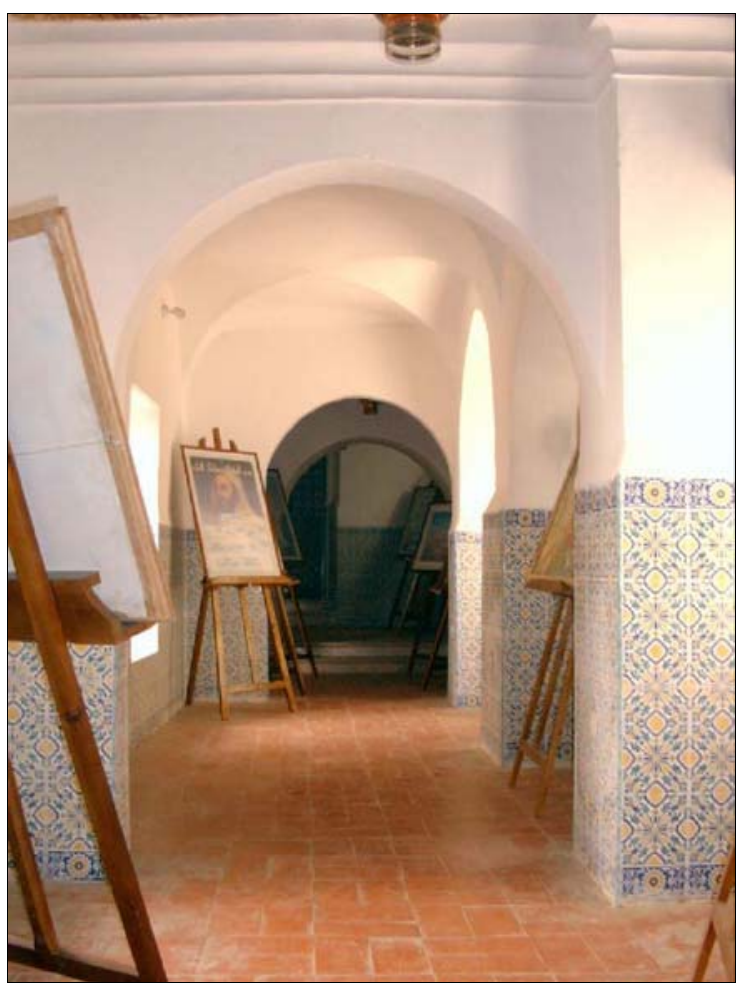




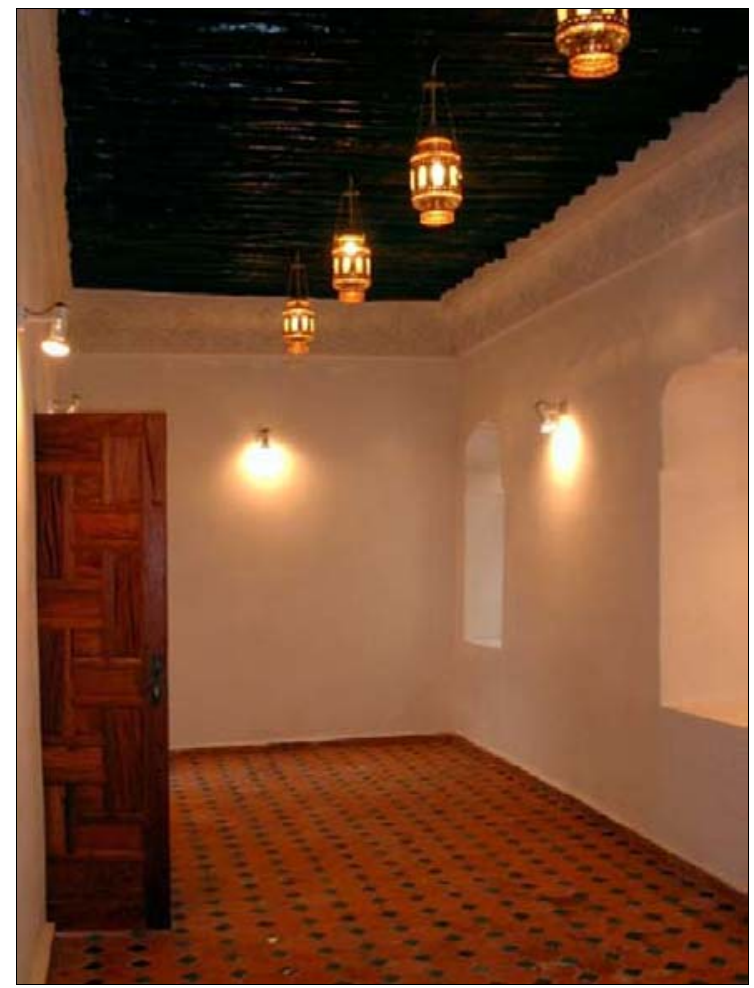

- Fig. 7. Une des salles du tribunal. L'ambiance d'époque semble retrouvée (M. Bouziane).

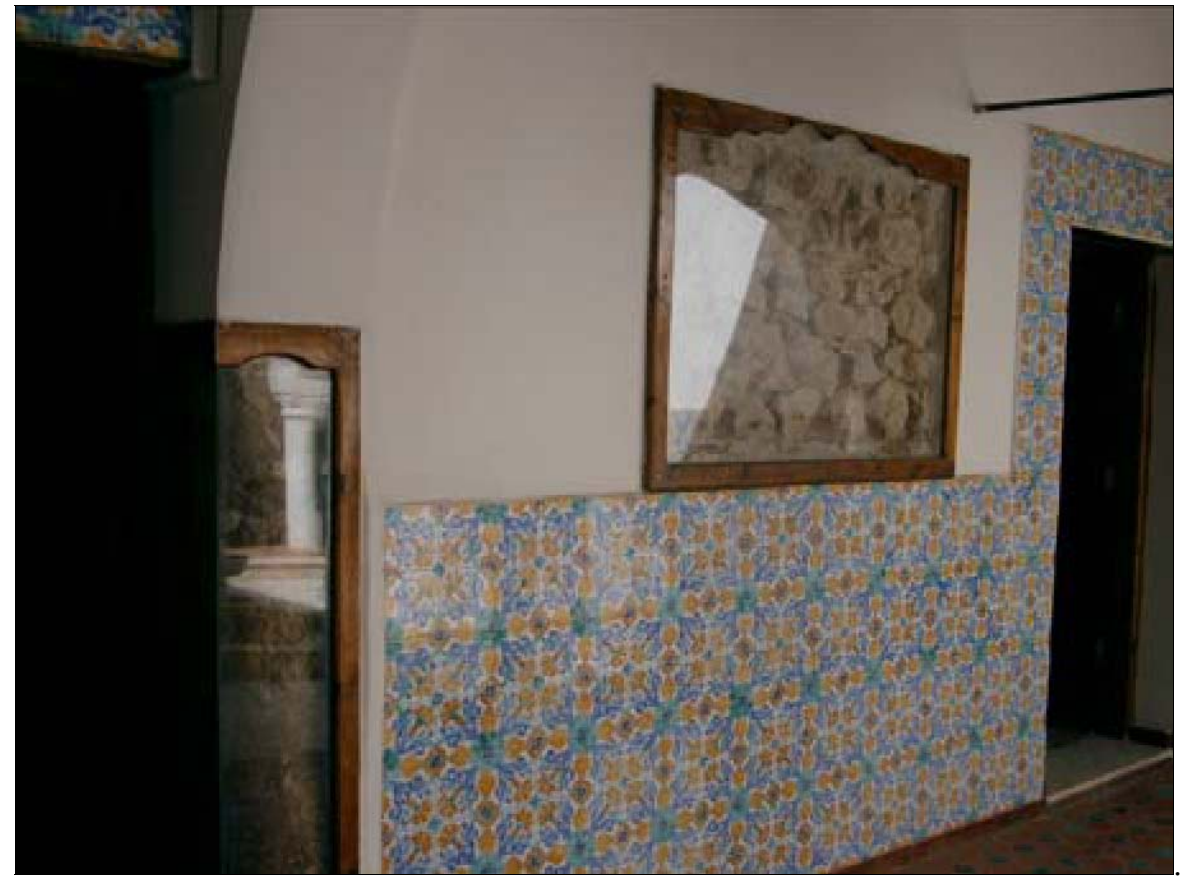

- Fig. 8. Nouveau concept en Algérie; L’histoire comme spectacle. Patio du Tribunal (M. Sadeg). 